\title{
Iris Pattern Recognition
}

\author{
Dr. S. T. Deepa ${ }^{1}$,Praneetha $V^{2}$. \\ ${ }^{2}$ Asst. Professor / HOD Department Of Compter Science Shri S. S. Shasun Jain College, Chennai \\ ${ }^{I}$ M.Phil. Research Scholar Department Of Compter Science Mother Teresa Women's University, Chennai
}

\begin{abstract}
The earlier experimental which were conducted has resulted in the biometric templates, which include the iris of human eye and the attributes of the individual, that are produced using different methods of recognition, which can be matched with the help of central rays in the convex polyhedral cones and that these templates are protected by a method which is extended from Iris-templates and it can be broken into. The results of these experiments clearly show that, the convex polyhedral cone template cannot be presumed secure without a thorough security analysis. If there is a contact lens present then it imposes challenge in the recognition of the iris pattern. It is more complicated when the lens is a textured lens used as cosmetics. These types of lenses are readily available in the market. This study also intends at analyzing the influence of these constraints in the recognition of iris of the human eye. The approach that is being proposed overtakes the other types of algorithms used for detection and improves the performance of the recognition process of the iris. When it comes to those who wear a contact lens, the identification of the presence of a lens is the initial task which should be taken in order to increase the reliability and usability of the recognition process. The astonishing success of Iris-templates depends deeply on its computational benefits and it includes the enormously high matching speed for identification of iris on a large-scale and automatic threshold adjustment which is based on the quality of the image. Many techniques which were modified from Iris-templates were suggested for iris recognition and the recognition based on the individuals attributes.
\end{abstract}

Keywords: Biometrics, iris recognition, Edge detection, Gradient, Median filter

\section{Introduction}

The present study proposes a methods and techniques which can be applied in the recognition of patterns of the human iris in order to recognize an individual person. The study also discusses the method of transforming the images of the iris of human eye for a one dimensional space to a two dimensional spaced image. This overcomes the limitation of generation of a synthetic image using limited data. A recent stress on security issues has paved way for

more and more research coming up in the field of biometrics relating to the identification and verification of individuals.

The biometric feature of an individual is an innate physical quality or behavioral trait. This characteristic is unique among individuals and no two individuals will have the same attribute. The iris of human is considered to be an effective biometric feature for the identification of individuals. The iris of human eye is nothing but a coloured ring on the human eye and this lies in between the pupil of the eye and the white sclera. Each iris of human has a unique code called the Iris Code which has delicate features and they vary from one person to another. Features of iris remain constant throughout the lifetime of an individual. They do not change even as the individual ages. This is the main reason that the human iris is considered to be the most effective one for a highly accurate and effective identification systems. The uniqueness of iris texture lies in the fact that the processes generating those textures are completely chaotic but stable. Hence in order to use the iris as a biometric, the feature extraction should be able to capture and encode this randomness present in the iris texture.

The iris recognition system is classified into three broad categories based on a widespread survey of related literature. The classification based on the extraction of the features is: extraction based on appearance, extraction based on texture and extraction based on features.

\section{Related Work}

[1] IrisCode has been used to gather iris data for 430 million people. Because of the huge impact of IrisCode, it is vital that it is completely understood. This paper first studies the relationship between bit probabilities and a mean of iris images and then uses the Chi-square statistic, the correlation coefficient and a resampling algorithm to detect statistical dependence between bits. The results show that the statistical dependence forms a graph with a sparse and structural adjacency matrix. A comparison of this graph with a graph whose edges are defined by the inner product of the Gabor filters that produce IrisCodes shows that partial statistical dependence is induced by the filters and propagates through the graph. Using this statistical 
information, the security risk associated with two patented template protection schemes that have been deployed in commercial systems for producing application-specific IrisCodes is analyzed. To retain high identification speed, they use the same key to lock all IrisCodes in a database. The belief has been that if the key is not compromised, the IrisCodes are secure. This study shows that even without the key, application-specific IrisCodes can be unlocked and that the key can be obtained through the statistical dependence detected.

[2] A method of rapid visual recognition of personal identity is described, based on the failure of a statistical test of independence. The most unique phenotypic feature visible in a person's face is the detailed texture of each eye's iris: An estimate of its statistical complexity in a sample of the human population reveals variation corresponding to several hundred independent degree-of-freedoms. Morphogenetic randomness in the texture expressed phenotypically in the iris trabecular meshwork ensures that a test of statistical independence on two coded patterns originate from different eyes is passed almost certainly, whereas the same test is failed almost certainly when the compared codes originate from the same eye. The visible texture of a person's iris in a real-time video image is encoded into a compact sequence of multi-scale quadrature 2-D Gabor wavelet coefficients, whose most-significant bits comprise a 256-byte "iris code". Statistical decision theory generates identification decisions from Exclusive OR comparisons of complete iris codes at the rate of 4000 per second, including calculation of decision confidence levels. The distributions observed empirically in such comparisons imply a theoretical "cross-over" error rate of one in 131000 when a decision criterion is adopted that would equalize the false accept and false reject error rates. In the typical recognition case, given the mean observed degree of iris code agreement, the decision confidence levels correspond formally to a conditional false accept probability of one in about $10^{3}$.

[3] Algorithms developed by the author for recognizing persons by their iris patterns have now been tested in many field and laboratory trials, producing no false matches in several million comparison tests. The recognition principle is the failure of a test of statistical independence on iris phase structure encoded by multiscale quadrature wavelets. The combinatorial complexity of this phase information across different persons spans about 249 degrees of freedom and generates discrimination entropy of about $3.2 \mathrm{~b} \mathrm{~mm} 2$ over the iris, enabling real-time decisions about personal identity with extremely high confidence. The high confidence levels are important because they allow very large databases to be searched exhaustively (one-to-many "identification mode") without making false matches, despite so many chances. Biometrics that lacks this property can only survive one-to-one ("verification") or few comparisons. This paper explains the iris recognition algorithms and presents results of 9.1 million comparisons among eye images from trials in Britain, the USA, Japan, and Korea. The complete execution time of this 2-D focus assessment algorithm, implemented in $\mathrm{C}$ using pointer arithmetic, operating on a (480 640) image, is 15 min on a 300-MHz RISC processor.

[4] This paper presents the following four advances in iris recognition: 1) more disciplined methods for detecting and faithfully modeling the iris inner and outer boundaries with active contours, leading to more flexible embedded coordinate systems; 2) Fourier-based methods for solving problems in iris trigonometry and projective geometry, allowing off-axis gaze to be handled by detecting it and "rotating" the eye into orthographic perspective; 3) statistical inference methods for detecting and excluding eyelashes; and 4) exploration of score normalizations, depending on the amount of iris data that is available in images and the required scale of database search. Statistical results are presented based on 200 billion iris cross-comparisons that were generated from 632500 irises in the United Arab Emirates database to analyze the normalization issues raised in different regions of receiver operating characteristic curves. It is hoped that the new imageprocessing algorithms introduced in this paper using active contours, deviated gaze correction, and other improvements can help in achieving those requirements. A further advance that allows IrisCodes to be indexed by their collisions with substrings, thereby replacing the need for exhaustive search (which is time consuming in large databases) by instead almost instantaneous direct addressing with IrisCodes,

[5] IrisCode is an iris recognition algorithm developed in 1993 and continuously improved by Daugman. It has been extensively applied in commercial iris recognition systems. IrisCode representing an iris based on coarse phase has a number of properties including rapid matching, binomial impostor distribution and a predictable false acceptance rate. Because of its successful applications and these properties, many similar coding methods have been developed for iris and palmprint identification. However, we lack a detailed analysis of IrisCode. The aim of this paper is to provide such an analysis as a way of better understanding IrisCode, extending the coarse phase representation to a precise phase representation, and uncovering the relationship between IrisCode and other coding methods. The analysis demonstrates that IrisCode is a clustering algorithm with four prototypes; the locus of a Gabor function is a two-dimensional ellipse with respect to a phase parameter and can be approximated by a circle in many cases; Gabor function can be considered as a phasesteerable filter and the bitwise hamming distance can be regarded as a bitwise phase distance. The paper also discusses the theoretical foundation of the impostor binomial distribution. This analysis is used to develop a precise phase representation which can enhance accuracy. Finally, the paper relates IrisCode and other coding methods. IrisCode first appeared sixteen years ago yet to our knowledge this is the first paper to provide a 
detailed analysis of this method. The analysis made here makes a number of contributions. It presents a complete analysis of IrisCode, demonstrating that IrisCode is a clustering algorithm and that the locus of a Gabor function is on a two dimensional ellipse with respect to the phase parameter. It also proves the equivalent relationship between the bitwise hamming distance and bitwise phase distance and shows that Gabor function can be considered as a phase-steerable filter. It then uses these properties and this relationship to develop a precise phase representation algorithm. This algorithm inherits the properties of IrisCode including robustness against brightness and contrast variations and rapid matching based on bitwise operators and cyclic representation. The experiments have shown that given the same quality of preprocessing precise phase representation is more accurate than IrisCode. Precise phase representation is a flexible representation for balancing the tradeoff between matching speed and identification accuracy. This paper has also discussed theoretical issues regarding the binomial impostor distribution of IrisCode and other coding methods. Finally, using the filter generating function and the coding scheme defined in this study, showing the relationships between IrisCode and other iris and palmprint recognition coding methods.

[6] There is increasing interest in the development of reliable, rapid and non-intrusive security control systems. Among the many approaches, biometrics such as palmprints provide highly effective automatic mechanisms for use in personal identification. This paper presents a new method for extracting features from palmprints using the competitive coding scheme and angular matching. The competitive coding scheme uses multiple 2-D Gabor filters to extract orientation information from palm lines. This information is then stored in a feature vector called the competitive code. The angular matching with an effective implementation is then defined for comparing the proposed codes, which can make over 9,000 comparisons within 1s. In our testing database of 7,752 palmprint samples from 386 palms, we can achieve a high genuine acceptance rate of $98.4 \%$ and a low false acceptance rate of $3 \times 10^{-6} \%$. The execution time for the whole process of verification, including preprocessing, feature extraction and final matching, is $1 \mathrm{~s}$.

[7] Biometrics-based verification, especially fingerprint-based identification, is receiving a lot of attention. There are two major shortcomings of the traditional approaches to fingerprint representation. For a considerable fraction of population, the representations based on explicit detection of complete ridge structures in the fingerprint are difficult to extract automatically. The widely used minutiae-based representation does not utilize a significant component of the rich discriminatory information available in the fingerprints. Local ridge structures cannot be completely characterized by minutiae. Further, minutiae-based matching has difficulty in quickly matching two fingerprint images containing a different number of unregistered minutiae points. The proposed filter-based algorithm uses a bank of Gabor filters to capture both local and global details in a fingerprint as a compact fixed length FingerCode. The fingerprint matching is based on the Euclidean distance between the two corresponding FingerCodes and hence is extremely fast. We are able to achieve a verification accuracy which is only marginally inferior to the best results of minutiae-based algorithms published in the open literature. Our system performs better than a state-of-the-art minutiae-based system when the performance requirement of the application system does not demand a very low false acceptance rate. Finally, we show that the matching performance can be improved by combining the decisions of the matchers based on complementary (minutiae-based and filter-based) fingerprint information

\section{System Description}

Among all the iris recognition algorithms, the most powerful iris recognition algorithm is Daugman Algorithm. The present paper has an insight on the attribute of bit pair which is based on secured key along with its central ray which is a rough illustration of the original biometric signal. The central ray is an anticipated ray and it is also an optimum ray of an objective function on a group of distribution. The algorithm is a resultant of the geometric properties of a convex polyhedral cone. In the present system, the biometric feature is fundamentally used to identify the face, handprint, fingerprint, voice and so on of an individual. But these all also have an error false report. So in the proposed system iris is used by generating code. Iris is said to be an internal organ and that is visible externally. Iris has a unique feature, and it is unique for each individual. Iris recognition is identifying the person based on the image of their eyes. The patterns are perfect for identification of biometrics as they are hard to modify and extremely complex. It is detected that the pattern of iris patterns formed even before birth and the characteristics of human iris remain constant throughout an individual's lifetime. It is used to implement and analyze local intensity variation-based method. Algorithms and Techniques used in this paper are: Gray Scale Conversion, Canny Edge Detection, Pupil Detection, Normalization, Feature Extraction and Median Filter.

\section{Image Conversion:}

\section{Description Of Each Module}

Grayscale images are different from one-bit black and white images. These are imaging images with only the two colours, black and white. They are also referred to as bi-level images or binary images. The 
Grayscale images are made of many shades of monochromatic, which denotes that the chromatic variation are absent in the image. Grayscale images are the results obtained from measuring the intensity of light at each and every pixel in a single band of the electromagnetic spectrum. In these cases they are monochromatic proper only when a given frequency is captured. They can also be produced from a full colour image.

\section{Edge Detection:}

The fundamental tool of processing an image is the process of Edge. This is particularly is in the areas of feature recognition and feature abstraction. This target at recognizing points in a digital image at which the brightness of image changes sharply or has some discontinuities.

The edges that is extracted from a two dimensional image of a scene of three-dimension can be categorized as viewpoint dependent or viewpoint independent. An edge with viewpoint independent reflects essential properties of the three-dimensional objects. An edge with viewpoint dependent changes as the viewpoint changes, and reflects the geometry of the scene, Gradients at each pixel in the smoothed image. The algorithm works in five steps they are Smoothing performs blurring of the image to remove noise. Finding gradients, in which edges should be marked where the gradients of the image have large magnitudes. Nonmaximum suppression, in these only local maxima should be marked as edges. Double thresholding, the potential edges are determined by thresholding. Edge tracking by hysteresis where the final edges are determined by suppressing all edges that are not connected to a very strong edge.

\section{Pupil Detection:}

The iris image that has been acquired should to be preprocessed so as to detect the iris, which is an annular portion between the pupil of the human eye and the sclera. The first step in localization of the iris is to identify pupil which is the black circular part surrounded by iris tissues. The center of pupil can be used to detect the outer radius of patterns of iris. The steps involved in this process are: detection of Pupil and localization of the outer iris. Circular Hough Transformation can be used for pupil detection. The main idea of this procedure is to find curves that can be parameterized like straight lines, polynomials, circles, in a suitable parameter space. External noise is removed by blurring the intensity of the image. Care must be taken as too much blurring may dilate the boundaries of the edge or it may make it difficult to detect the outer boundary of the iris, separating the eyeball and sclera. Thus a special smoothing filter such as the median filter is used on the original intensity image. This type of filtering eliminates sparse noise while preserving image boundaries. After filtering, the contrast of image is enhanced to have sharp variation at image boundaries using histogram equalization.

\section{Normalization}

It is a necessity to remove blurred images before the features can be extracted. Localizing iris from an image outlines the annular portion from the rest of the image. The concept of rubber sheet modal suggested by Daugman takes into consideration the possibility of pupil dilation as it appears in different size in different images. For this purpose, the coordinate system is changed by unwrapping the iris and mapping all the points within the boundary of the iris into their polar equivalent. The mapped image has $80 \times 360$ pixels. It means that the step size is same at every angle. This normalization to an extent reduces the elastic distortions of the iris.

\section{Feature Extraction:}

The features for distinguishing two iris images can be extracted by making use of the corners in the normalized iris image. The corner point alone is not considered in order to extract the feature, but the $5 \times 5$ window is also taken to produce the texture with high quality. The steps involved in corner detection algorithm are as follows: In step1, the normalized iris image is used to detect corners using covariance matrix. Step 2 takes care that the detected corners between the database and query image are used to find cross correlation coefficient. In step3 if the number of correlation coefficients between the detected corners of the two images is greater than a threshold value then the candidate is accepted by the system.

\section{Matching:}

The given two iris images are determined to be of the same class when they are compared of the feature vectors, using a Daugman like X-OR operation. The matching of iris would be done using this procedure. The matching would be done with the trained images. The matching score is calculated and if the score is high it indicates that the images are matches and if the score is low it indicated that the images did not match. If the images matches and present in the database then it further shows the details of that individual. If it is not matched with the database, then the details will be collected for further investigation, as per the requirement. 


\section{System Architecture}

The system architecture describes about the scanned iris image which includes the following techniques and algorithm. The gray scale conversion is the conversion of a colorful iris image to a monochromatic gray and white image. This module includes median filter which helps in extracting noises such as eye lashes, change in white area around pupil. Canny edge detection is next algorithm technique. This detection algorithm consists of three different phases such as smoothing, finding gradients and threshold value. Pupil detection algorithm is used to find the inner and outer circle radius. It has two types such as improved half transform and localization which is used to find the curves. Another type is Standard half transform which is used to find the straight lines. Normalization is the algorithm. It is used to minimize the dimensional inconsistencies between iris regions. Feature extraction is the algorithm which helps us to identify the most important and minute differences in an iris image

\section{Input Image:}

\section{Experimental Results}

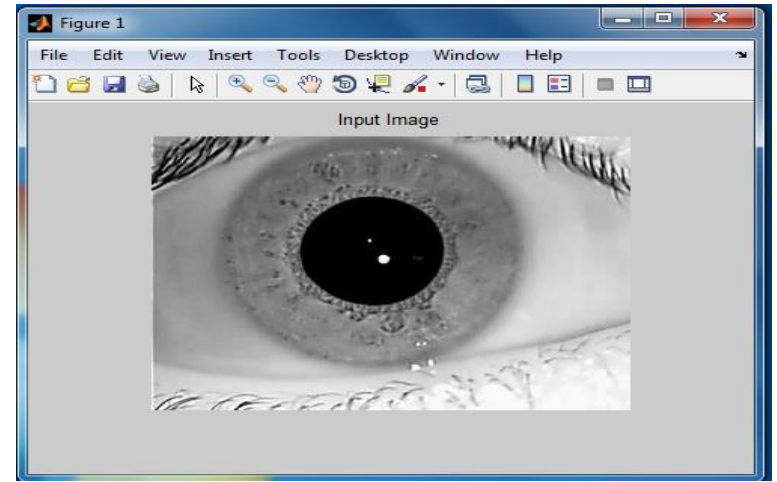

\section{Conversion of Image:}

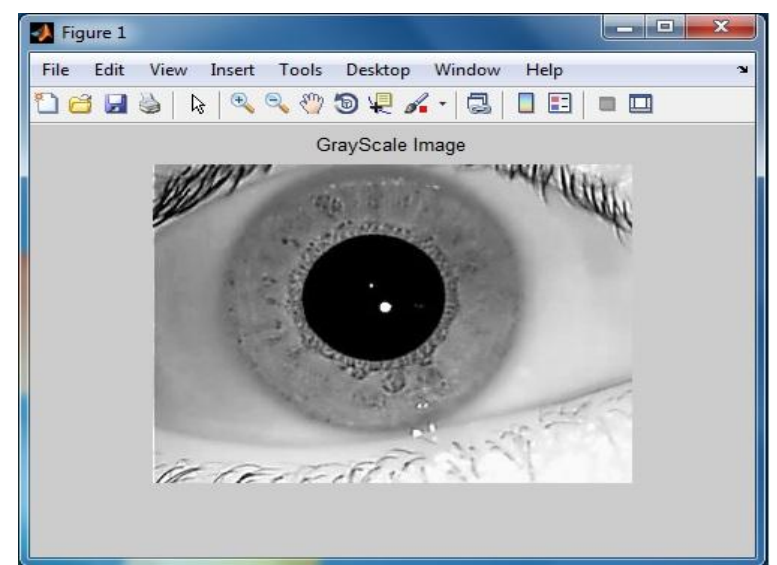

\section{Detection of Edge:}

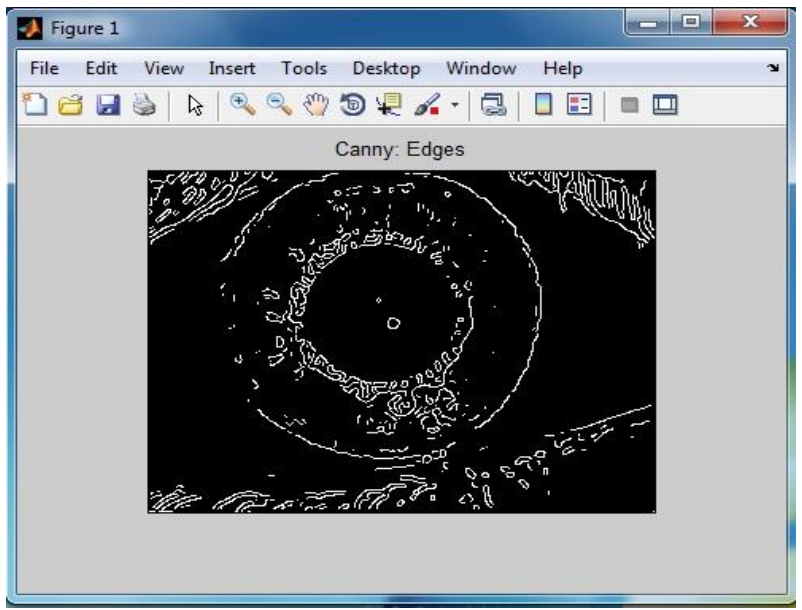



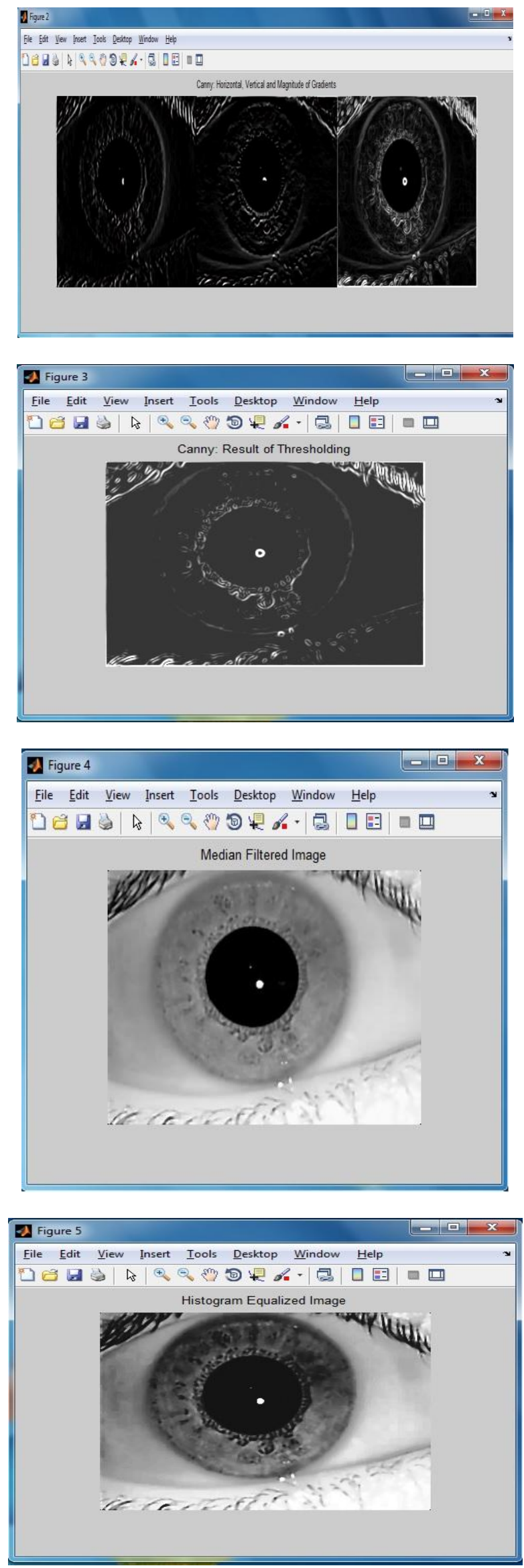


\section{Detection of Pupil:}
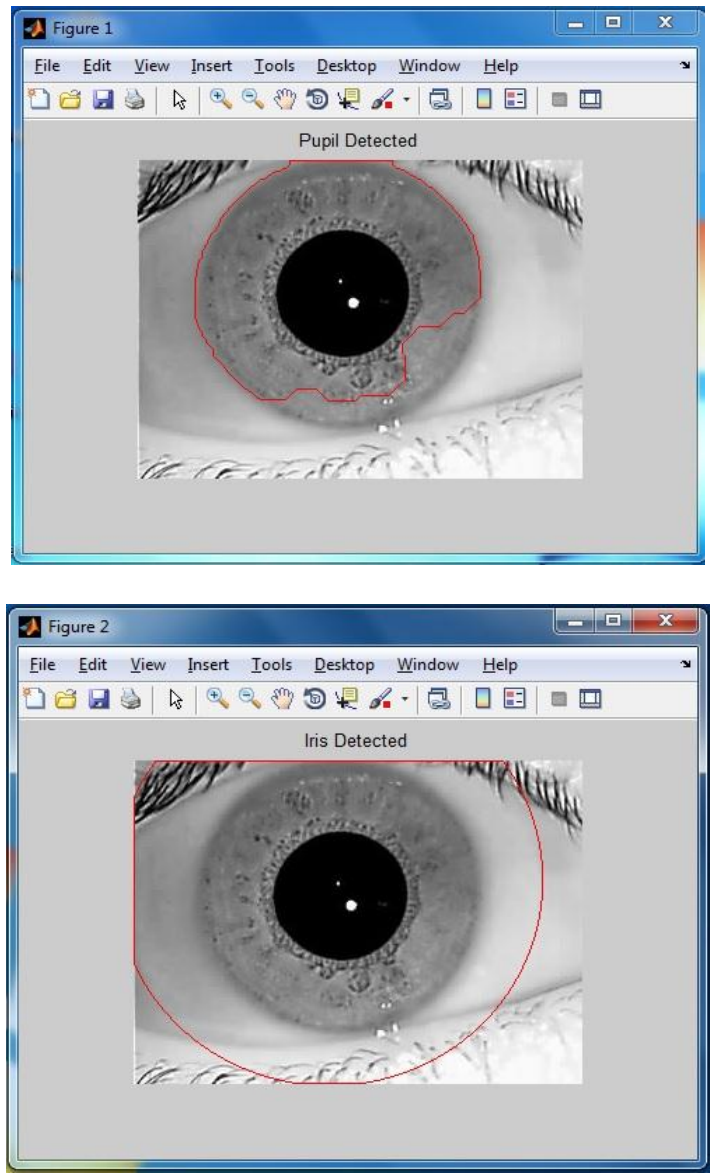

\section{Normalization:}

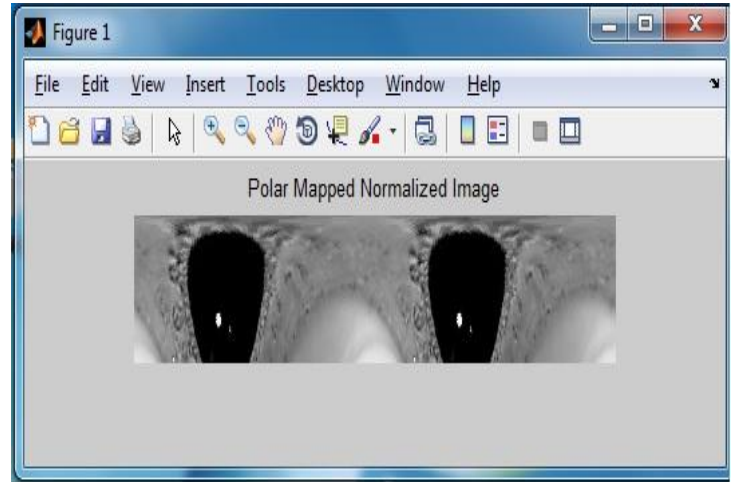

\section{Extraction of Features:}

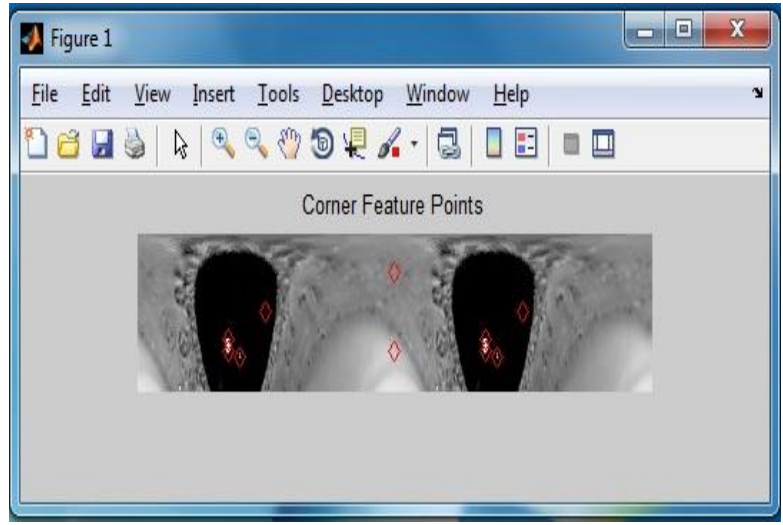




\section{Iris Recognition:}

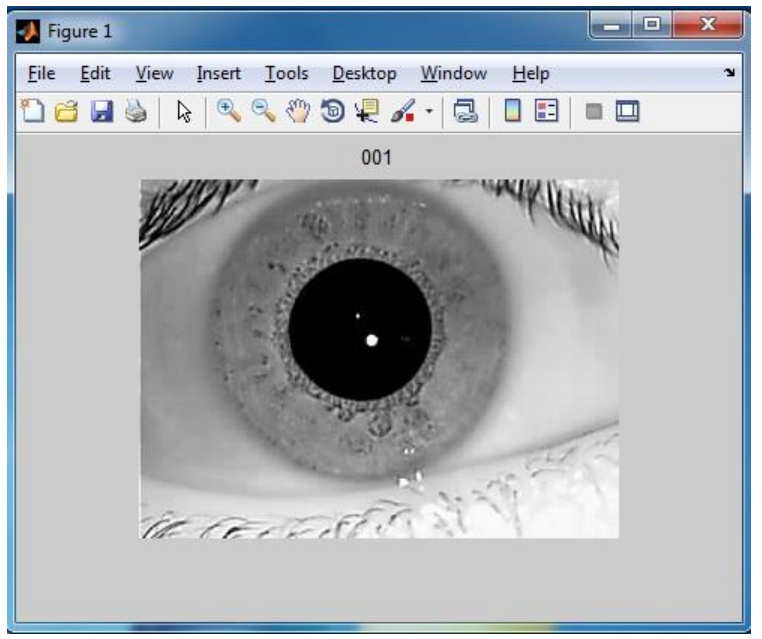

\section{Matching Of Two Irises:}

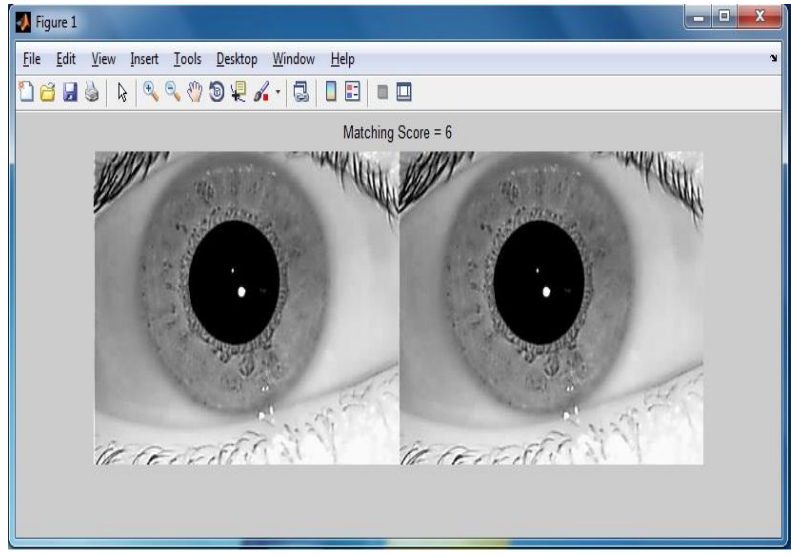

\section{Conclusion And Further Enhancement}

In the present work, the method for generating an iris code, which is embedded in the natural texture of human eye for an individual, is discovered. When the generated textures are used in recognition of iris system, it would produce a response which will be related to the original texture of the iris. No work presently exists that encompasses the modeling the iris from the bit code of iris, which is normally considered to be an unrecognizable data. The expected result of proposed system will provide the unique identification to the user. Efficiency will be more in the authentication. It will be more secure while accessing bank accounts and also in the hospitals for the patients details. Duplication will not be there in the authentication.

In the present work, the iris texture is generated which starts from just the iris bit code of an individual and it has been embedded with the necessary texture within any texture of an iris to produce an iris code. The main benefit of this technique is that the alternate texture of iris can be created and it will give a very comparable iris code when matched to the original iris texture. As future work, the exploration would be on the countermeasure for identifying such spoof endeavors.

\section{References}

[1]. A. W. K. Kong, “A Statistical Analysis of IrisCode and Its Security Implications,” IEEE Trans. Pattern Anal. Mach. Intell.,vol. 37, no. 3, pp. 513-528, Mar. 2015.

[2]. J. G. Daugman, "High confidence visual recognition of persons by a test of statistical independence," IEEE Trans. Pattern Anal. Mach. Intell.,vol. 15, no. 11, pp. 1148-1161, Nov. 1993.

[3]. J. Daugman, "How iris recognition works," IEEE Trans. Circuits Syst.Video Technol., vol. 14, no. 1, pp. 21-30, Jan. 2004.

[4]. J. Daugman, "New methods in iris recognition," IEEE Trans. Syst. Man, Cybern. B, Cybern., vol. 37, no. 5, pp. 1167-1175, Oct. 2007.

[5]. A. W. K. Kong, D. Zhang, and M. Kamel, “An analysis of IrisCode,’IEEE Trans. Image Process., vol. 19, no. 2, pp. 522-532, Feb. 2010 .

[6]. A. W. K. Kong and D. Zhang, "Competitive coding scheme for palmprint verification," in Proc. Int. Conf. Pattern Recognit., vol. 1. 2004, pp.520-523.

[7]. A. K. Jain, S. Prabhakar, L. Hong, and S. Pankanti, "Fiterbank-based fingerprint matching," IEEE Trans. Image Process., vol. 9, no. 5, pp. 846-859, May 2000. 